Research Article

\title{
An experimental study of a novel combination of a herbal drug with an allopathic drug to evaluate the antihyperglycemic effect of irbesartan plus curcumin and comparison with glibenclamide
}

\author{
Sharad Tularam Leve, Himani Banga, Pratap Shankar*, Rakesh Kumar Dixit
}

Department of Pharmacology and Therapeutics, King George's Medical University, Lucknow226003, India

Received: 22 January 2013

Accepted: 27 January 2013

*Correspondence to:

Dr. Pratap Shankar,

Email: pratap.mbi@gmail.com

(C) 2013 Leve ST et al. This is an open-access article distributed under the terms of the Creative Commons Attribution License, which permits unrestricted use, distribution, and reproduction in any medium, provided the original work is properly cited.

\begin{abstract}
Background: Curcumin has been used as a traditional remedy for many diseases in Ayurveda. Many studies have proved antidiabetic and antihyperlipidimic effects of curcumin. The present study was conducted to evaluate antihyperglycemic actions of Irbesartan in combination with Curcumin.

Methods: After baseline sampling, 18 rats of diabetic groups i.e. Group 1 to Group 3, were started with exclusive high fat diet and water ad libitum for next 4 weeks. At the end of 4 weeks, each rat was given Inj. Streptozotocin in a dose of $30 \mathrm{mg} / \mathrm{kg}$ body weight, dissolved in $0.2 \mathrm{ml}$ of citrate buffer $(\mathrm{pH} 4.5)$ intraperitoneally. Drugs were given orally in normal saline by gastric gavage with the help of rat feeding cannula.

Results: Rats of diabetic group 2, receiving combination of Irbesartan 20mg/kg and Curcumin $200 \mathrm{mg} / \mathrm{kg}$ body weight for 6 weeks, have shown significant decrease is mean FPG level (FPG- 29.69\% reduction, $p<0.001$ ). Irbesartan+Curcumin had no any hypoglycemic effect in normoglycemic rats as well as diabetic rats.

Conclusions: Irbesartan in combination with Curcumin has significant antihyperglycemic effects. At the same time, Irbesartan combined with Curcumin, does not produce hypoglycemia in normal as well as diabetic rats.
\end{abstract}

Keywords: Curcumin, Irbesartan, Glibenclamide, Antihyperglycemic, Diabetes mellitus

\section{INTRODUCTION}

Diabetes mellitus describes a metabolic disorder of multiple etiologies characterized by insulin resistance, relative insulin deficiency and hyperglycemia with disturbances of carbohydrate, fat and protein metabolism. ${ }^{1}$ The goal for treatment of diabetes is to prevent its acute manifestations and long-term microvascular and macrovascular complications. Medicinal herbs with antihyperglycemic activities are increasingly sought after by diabetic patients and health care professionals. Commonly used herbs and other alternative therapies, are considered less likely to have the side effects of conventional treatments for NonInsulin Dependent Diabetes Mellitus (NIDDM). ${ }^{2}$

A recent study involving 2055 patients in the US also reveals that the consumption pattern of traditional medications has no significant gender or social difference. $^{3,4}$ Herbal products are made of complex mixture of pharmacologically active phytochemicals ${ }^{5}$, most of which are secondary metabolites generated through the shikimate, acetate-malonate, and acetatemevalonate path- ways. These constituents include phenolics (such as tannins, lignins, quinolones, and salicylates), phenolic glycosides (such as flavonoids, cyanogens, and glucosinolates), terpenoids (such as sesquiterpenes, steroids, carotenoids, saponins, and iridoids), alka- loids, peptides, polysaccharides (such as gums and mucilages), resins, and essential oils which often contain some of the afore- mentioned classes of phytochemicals. ${ }^{6,7}$ This complexity increases the risk of clinical drug interactions. ${ }^{3}$

Curcumin is an extract of herb Curcuma longa i.e. turmeric, commonly used spice. It has been used as a traditional remedy for many diseases in Ayurveda. Many 
studies have proved antidiabetic and antihyperlipidimic effect of curcumin.

Irbesartan is an Angiotensin II AT1 receptor blocker (ARB). It is largely being used in treatment of hypertension and hypertension-related cardiovascular end-organ damage. Some recent studies have shown that, it possesses antihyperglycemic action. Few studies have shown that it has PPAR-alpha agonist action also. Hence it can have antihyperlipidemic action also. It also decreases the inflammatory cytokines like IL 1b, IL6, TNF alpha etc responsible for metabolic syndrome. But further studies are needed, that's why this drug has been selected. In our study, we have tried to combine one allopathic drug with a herbal drug and evaluated the effect of curcumin on antihyperglycemic actions of Irbesartan. ${ }^{8}$

\section{METHODS}

The study was conducted in the Department of Pharmacology and Therapeutics, King George's Medical University, Lucknow (Erstwhile Chhatrapati Shahuji Maharaj Medical University). Prior permission was sought from the Institutional Animal Ethics Committee for conducting the study.

\section{Experimental animals and rearing conditions}

24 adult healthy male Wistar rats, of similar physical constitution (in terms of age, body weight), weighing 160-200 gm had been used in study. Animals had been obtained from CPCSEA certified animal house [Indian Institute Of Toxicology Research, Lucknow (IITR)]. They were given standard pellet diet and water ad libitum and were kept in Institutional animal house under temperature, humidity and light and dark cycle-controlled environment $\left[25 \pm 2{ }^{\circ} \mathrm{C}, 70 \%, 12 \mathrm{hrs}\right.$ ' cycle). The animals were housed for two weeks prior to the experiments to acclimatize to new environment. The maintenance of the animals and the experimental procedures were in accordance with the guiding principles of Institutional Animal Ethics committee and the 'Guide for the Care and Use of Laboratory Animals', National Research Council, 1996 (Latest revision in 2011). The guidelines of Committee for the Purpose of Control and Supervision of Experiments on Animals (CPCSEA), Govt. of India were followed.

\section{Drugs and chemicals}

Following drugs and other materials were used in this study. Drugs were given orally in normal saline by gastric gavage with the help of rat feeding cannula and streptozotocin intraperitoneally.

1) Tablet Irbesartan: Dose - $20 \mathrm{mg} / \mathrm{kg}$ body weight. Dose was calculated from human dose (Paget and Barnes, 1964); purchased from government authorized medical store.
2) Curcumin: Extract, Dose - $200 \mathrm{mg} / \mathrm{kg}$ body weight. ${ }^{8}$ Eva Green Curcumin, (manufactured by Sir Biotech India.) It was purchased from Eva Lifestyle Private Ltd, Delhi.

3) Tablet Glibenclamide: Dose - $0.3 \mathrm{mg} / \mathrm{kg}$ body weight, purchased from government authorized medical store. ${ }^{9}$

4) Injection Streptozotocin: Dose - 30mg/kg body weight in $0.2 \mathrm{ml}$ of citrate buffer ( $\mathrm{pH} 4.5) .{ }^{10}$ It was purchased from Merck India Pvt. Ltd.

5) High Fat Diet: High fat diet was purchased from Dayal Industries Pvt. Ltd., Barabanki Road, Lucknow, Uttar Pradesh; with composed of Crude Fat (Prepared from Rice Bran) (15\%); Crude Protein (16\%); Acid Insoluble Ash (2.30\%); Moisture (8\%); Vitamins and Minerals appropriate quantity.

\section{Experimental design}

The present study had been designed to evaluate the antihyperglycemic action of combination of Irbesartan+Curcumin and to compare this with that of standard drug, Glibenclamide. It was also studied whether this combination caused hypoglycaemia as a side effect in normoglycemic rats. 24 adult male albino wistar rats, randomly divided in four groups of 6 rats each, were used. Group 1 to 3 were diabetic groups. Group 4 (Hypoglycemic group) was used to study the hypoglycemic effect of combination of Irbesartan plus Curcumin in normoglycemic rats. After the acclimatization of 2 weeks, study was started. At day 0 (Week 0), fasting blood samples were taken from each rat from retro-orbital venous plexus under general anaesthesia using Pentobarbitone. Fasting plasma glucose levels were evaluated using the Glucose-Oxidase (GOD) Peroxidase (POD) Method.

\section{Induction of Diabetes Mellitus Type 2}

After baseline sampling, 18 rats of diabetic groups i.e. Group 1 to Group 3, were started with exclusive high fat diet and water ad libitum for next 4 weeks. At the end of 4 weeks, each rat was given Inj. Streptozotocin in a dose of $30 \mathrm{mg} / \mathrm{kg}$ body weight ${ }^{10}$, dissolved in $0.2 \mathrm{ml}$ of citrate buffer ( $\mathrm{pH}$ 4.5) intraperitoneally. One week after Streptozotocin injection i.e. at week 5, fasting plasma glucose level were studied again. Rats with fasting blood sugar level more than $150 \mathrm{mg} / \mathrm{dl}$ were considered diabetic. ${ }^{11}$ They were continued on high fat diet ad libitum for remaining duration.

\section{Animal grouping}

Group 1: Diabetic control Group- No test drugs were given, only normal saline $1 \mathrm{ml}$ was given orally daily and were fed with high fat diet for 6 weeks.

Group 2: Combination of Irbesartan plus Curcumin Group $(I+C)$ - These 6 rats were given Irbesartan $20 \mathrm{mg} / \mathrm{kg}+$ Curcumin $200 \mathrm{mg} / \mathrm{kg}$ body weight dissolved in 
normal saline, orally daily and high fat diet for 6 weeks. $^{8,12}$

Group 3: Standard treatment group- These rats were given Glibenclamide $0.3 \mathrm{mg} / \mathrm{kg}$ body weight, dissolved in normal saline, orally daily and high fat diet for 6 weeks. ${ }^{9}$

Group 4: Irbesartan+Curcumin Hypoglycemic GroupThey received normal diet + Irbesartan 20mg/kg + Curcumin $200 \mathrm{mg} / \mathrm{kg}$ body weight, dissolved in normal saline, orally daily for 6 weeks.

At completion of treatment i.e. at $11^{\text {th }}$ weeks, fasting plasma glucose levels were again evaluated.

\section{Statistical analysis}

A one-sample Kolmogorov-Smirnov test was used to investigate whether the variables were normally distributed. The one way analysis of variance (ANOVA) was used to assess the comparability of the groups assigned to the treatment groups. Independent $t$ test/Tuke's pairwise comparison was used to compare the FPG levels between 2 treatment groups. Statistical significance was based on a two-tailed $p$ value $<0.05$.

\section{RESULTS}

The effects of high fat diet (HFD) and different drugs (Group 1: Control, Group 2: Irbesartan+Curcumin, Group 3: Standard- Glibenclamide) on FPG have been summarized in the Table 1. The FPG level in Group 1 was $79.18 \pm 6.90$ at week 0 which significantly increased to $199.96 \pm 19.44$ at week $5(p<0.001), 220.70 \pm 20.07$ at week $11(p<0.001)$. The increase in FPG level from Week 5 to Week 11 was also statistically significant $(p<0.001)$. The FPG level in Group 2 was significantly increased from Week $0 \quad(74.89 \pm 8.64)$ to week 5 $(190.61 \pm 18.55, \quad p<0.001)$, week $11 \quad(133.87 \pm 11.39$, $p<0.001)$ and decreased from week 5 to week 11 $(p<0.001)$. Almost similar observation was found for Group 3.

The Table-2 depicts the average percentage change in FPG level from week 0 to follow-ups. There was increase in the level of FPG in all the groups from week 0 to 5 and week 11. However, higher percentage decrease was observed in Group $3(-45 \%)$ as compared to Group 2 ($29.69 \%$ ), from week 5 to week 11 . There was $10.44 \%$ increase in the level of FPG in Group 1.

Table 1: Effect of combination of Irbesartan+Curcumin \& Glibenclamide on FPG levels.

\begin{tabular}{|lllllll|}
\hline \multirow{2}{*}{ Groups } & \multicolumn{3}{c|}{ FPG level (Mean \pm SD) } & \multicolumn{3}{c|}{$p$-value } \\
& Week 0 & Week 5 & Week 11 & Week0 vs week 5 & Week0 vs week11 & Week5 vs week 11 \\
\hline Group 1 & $79.18 \pm 6.90$ & $199.96 \pm 19.44$ & $220.70 \pm 20.07$ & $<0.001^{*}$ & $<0.001^{*}$ & $<0.001^{*}$ \\
\hline Group 2 & $74.89 \pm 8.64$ & $190.61 \pm 18.55$ & $133.87 \pm 11.39$ & $<0.001^{*}$ & $<0.001^{*}$ & $<0.001^{*}$ \\
\hline Group 3 & $76.78 \pm 9.46$ & $191.22 \pm 28.08$ & $105.17 \pm 15.44$ & $<0.001^{*}$ & $<0.001^{*}$ & $<0.001 *$ \\
\hline
\end{tabular}

* Statistically significant

Table 2: Mean percent change in FPG level.

\begin{tabular}{|c|c|c|c|}
\hline \multirow{2}{*}{ Groups } & \multicolumn{3}{|c|}{ Mean \% change in FPG level } \\
\hline & Week 0 to week 5 & Week 0 to week 11 & Week 5 to week 11 \\
\hline Group 1 & 152.85 & 179.27 & 10.44 \\
\hline Group 2 & 155.24 & 79.48 & -29.69 \\
\hline Group 3 & 149.23 & 37.07 & -45.00 \\
\hline
\end{tabular}

\section{Evaluation of hypoglycemic effect}

Rats of Group 4 were used to study hypoglycemic effects of combination of Irbesartan plus Curcumin in normoglycemic rats. They were given normal pellet diet and water ad libitum and kept in similar conditions. Along with this, Group 4 was treated with Irbesartan $20 \mathrm{mg} / \mathrm{kg}+$ Curcumin $200 \mathrm{mg} / \mathrm{kg}$ body weight daily for 6 weeks. $^{8,12}$ After 6 weeks, their fasting plasma glucose levels were evaluated again. No disease was induced in them.

\section{DISCUSSION}

Insulin resistance and $\beta$ cell dysfunction are two main components of type 2 DM. Type 2 diabetes is characterized by progressive deterioration of normal pancreatic $\beta$ cells function. In $\beta$ cells, oxidative glucose metabolism leads to production of reactive oxygen species, which are normally detoxified by catalase and superoxide dismutase enzymes. Persistent hyperglycemia leads to generation of large quantity of reactive oxygen species in these cells. These reactive oxygen species may subsequent damage to cellular components. ${ }^{13}$ In diabetic, and non- diabetic obese patients, enhanced adipocyte lipolysis raises the FFA [also known as non esterified fatty acid (NEFA)]. In the presence of glucose, fatty acid oxidation in $\beta$ cells is inhibited and accumulation of long chain acyl co-A occurs. ${ }^{14}$ Long chain acyl co-A can itself diminish insulin secretory process by either opening $\beta$ cell potassium channels; or increased expression of uncoupling protein - 2 which would lead to reduced ATP formation and hence decreased insulin secretion. Other possible mechanisms for reduced secretion of insulin may be by apoptosis of $\beta$ cells, possibly via fatty acid or triglyceride induced ceramide synthesis or generation of nitric oxide. Janson suggested that small aggregates of 
Amylin (an islet amyloid polypeptide), which is cosecreted with insulin, are cytotoxic to $\beta$ cells. ${ }^{15}$ Amylin aggregates may be further responsible for pathophysiological processes of DM.

The present study was conducted to evaluate antihyperglycemic actions of Irbesartan combination with Curcumin. The results of test drugs groups were compared with vehicle control treated group as well as standard drugs treatment group. Glibenclamide were chosen standard treatment drugs for hyperglycemia. Whether Irbesartan in combination of Curcumin had any hypoglycemic action in normal rats, was also studied. The galore of studies have proved the efficacy of herb Curcuma longa, especially its active constituent Curcumin, in management of DM, dyslipidemia as well as inflammation associated with metabolic syndrome. The diabetes model used was, high fat diet - low dose Streptozotocin induced type 2 DM in adult albino wistar rats. We have chosen the oral route for administering the drugs, as this route is natural, most common and acceptable route for drug administration. In literature, Albino wistar rats have been very commonly used in innumerable studies of diabetes. They were easily available from CPCSEA certified animal house. Hence they were chosen for study.

\section{Hypoglycemic effect}

No disease was induced in these rats and they were fed normal pellet diet ad libitum for 6 weeks. After evaluation of FPG levels at day 0 , group 4 received combination of Irbesartan $20 \mathrm{mg} / \mathrm{kg}$ and Curcumin $200 \mathrm{mg} / \mathrm{kg}$ body weight for 6 weeks. FPG levels were again evaluated. The decrease in FPG level in group 4 (from $86.03 \pm 13.41$ $\mathrm{mg} / \mathrm{dl}$ to $85.46 \pm 10.19 \mathrm{mg} / \mathrm{dl}$ ), was not statistically significant $(p>0.05)$. In diabetic groups also, Irbesartan+Curcumin didn't lower the FPG levels of diabetic rats below normal levels i.e. they didn't cause hypoglycemia. Hence, Irbesartan+Curcumin had no any hypoglycemic effect in normoglycemic rats as well as diabetic rats. These results of Curcumin treated groups are consistent with the previous study. ${ }^{16}$ However no study is reported regarding hypoglycaemic effect of Irbesartan in normoglycemic rats. The insulin as well as many commonly used oral hypoglycemic agents like sulfonylureas, meglitinides etc. can cause moderate to severe hypoglycemia, even life threatening; which limits their use or demand their cautious use. ${ }^{17-20}$ Hence, with respect to hypoglycaemic side effect, test drugs of present study seem to be safer.

\section{Combination (Irbesartan+Curcumin) treated group}

Rats of diabetic group 2, receiving combination of Irbesartan $20 \mathrm{mg} / \mathrm{kg}$ and Curcumin $200 \mathrm{mg} / \mathrm{kg}$ body weight for 6 weeks, have shown significant decrease is mean FPG level (FPG- 29.69\% reduction, significant $(p<0.001)$; table $1 \& 2$. Reduction was significant when compared with diabetic control. It is clear that combination of both is more efficacious in reducing raised FPG level in diabetic rats. Further animal and human studies are needed to support and reinforce our opinion about Irbesartan combination with Curcumin. Irbesartan in combination with Curcumin can be used very effectively to treat type $2 \mathrm{DM}$ and associated dyslipidemia and hypertension if found effective in clinical studies.

Results of standard drugs-group (Glibenclamide $0.3 \mathrm{mg} / \mathrm{kg}$ ) have shown more significant decrease in mean FPG (tables 1 \& 2) when compared with diabetic control, Irbesartan+Curcumin. Standard drugs have been used in such doses that they may produce appropriate result with maximal efficacy.

Hence, Irbesartan in combination with Curcumin has significant antihyperglycemic effects. At the same time, Irbesartan combined with Curcumin, does not produce hypoglycemia in normal as well as diabetic rats. Limitations of present study were as the serum insulin level was not measured. So level of Insulin resistance [Homeostasis Model Assessment of Insulin Resistance (HOMA-IR)] could not be estimated. Various mechanisms by which Irbesartan and Curcumin act can lead to decrease Insulin resistance. We could not measure the levels of cytokines like TNF, IL 6 , IL $1 \beta$ which are found to be associated with metabolic syndrome. Further studies can be planned using this animal model and including measurement of insulin levels and cytokines levels to strengthen the findings of present study.

\section{CONCLUSION}

The present study has been conducted with prime aims and objectives to evaluate the antihyperglycemic effects of Irbesartan in combination with Curcumin. Second part of study was to compare these effects with those of standard antihyperglycemic drug Glibenclamide. Third part of study was to evaluate the hypoglycemic effect of test drugs in normoglycemic rats. From the observations in the present study, following conclusions were drawn: Low dose Streptozotocin (30mg/kg body weight) injection after exclusive high fat diet $(15 \%)$ for 4 weeks, produces type 2 diabetes in all rats. Mortality after Streptozotocin at this dose is almost negligible; Percentage reduction in elevated FPG levels by Irbesartan+ Curcumin significant; Combination of Irbesartan plus Curcumin does not cause hypoglycaemia in normal as well as in diabetic rats. Antihyperglycemic actions of Irbesartan+Curcumin is less when compared with standard treatment drugs i.e. Glibenclamide.

In light of the above evidences, it can be concluded that Irbesartan (ARB) primarily an antihypertensive drug also possesses antihyperglycemic actions. Curcumin, a polyphenol present in frequently used household Indian herb Haldi/turmeric (Curcuma longa) for number of ailments including hepatobiliary disorders, has also shown to possess the antihyperglycemic actions. Combination treatment of Irbesartan with Curcumin can 
be used as antihyperglycemic. Further important finding of present study is that, Irbesartan+Curcumin do not produce hypoglycemia in normal as well as diabetic rats. The hypoglycemic effect in normal and diabetic rats was also not seen by combination treatment with Irbesartan and Curcumin. However this study needs further strength by doing animal studies and clinical trials before it can be implemented in therapeutics.

Funding: No funding sources

Competing interests: None declared

Ethical approval: The study was approved by the Institutional Animal Ethics Committee

\section{REFERENCES}

1. Powers AC, D'Alessio D. Endocrine Pancreas and Pharmacotherapy of Diabetes Mellitus and Hypoglycemia. In: Goodman \& Gilman's The Pharmacological Basis of Therapeutics. 12th ed., Brunton L, Chabner B, Knollman BB, editors. Mc. Graw Hill, New York, 2011:1237-73.

2. Gerstein HC, Miller ME, Byington RP, Goff DC, Jr., Bigger JT, Buse JB, et al. (2008). Effects of intensive glucose lowering in type 2 diabetes. $\mathrm{N}$ Engl J Med 2008;358:2545-59.

3. Fasinu PS, Bouic PJ, Bernd R. An overview of the evidence and mechanisms of herb-drug interactions. Front Pharmacol 2012;3:69.

4. Kessler RC, Davis RB, Foster DF, Van Rompay MI, Walters EE, Wilkey SA, Kaptchuk TJ, Eisenberg DM. Long-term trends in the use of complementary and alternative medical therapies in the United States. Ann Intern Med 2001;135:262-8.

5. Mok DKW, Chau F. Chemical information of Chinese medicines: a challenge to chemist. Chemometrics Intell Lab Syst 2006;82:210-7.

6. Wang JF, Wei DQ, Chou KC. Drug candidates from traditional Chinese medicines. Curr Top Med Chem 2008;8:1656-65.

7. Wills RB, Bone K, Morgan M. Herbal products: active constituents, modes of action and quality control. Nutr Res Rev 2000;13:47-77.

8. Nurullahoğlu-Atalik KE, Okudan N, Belviranli M, Gökbel H, Simşek L. Curcumin increases vasodilatory effect of cilostazol in diabetic rat aorta. Indian J Exp Biol 2012;50:128-32.

9. Gupta R, Gupta RS. Effect of pterocarpus marsupium in Streptozotocin-induced hyperglycemic state in rats: comparison with glibenclamide. Diabetol Croat 2009;38:39-45.

10. Wang HJ, Jin YX, Shen W, Neng J, Wu T, Li YJ, Fu ZW. Low dose streptozotocin (STZ) combined with high energy intake can effectively induce type 2 diabetes through altering the related gene expression. Asia Pac J Clin Nutr 2007;16 Suppl 1:412-7.

11. Mohammed A, Adelaiye AB, Abubakar MS, Abdurahman EM. Effects of aqueous extract of Ganoderma lucidum on blood glucose levels of normoglycemic and alloxan induced diabetic wistar rats. J Medicinal Plants Res 2007;1:34-7.

12. Paget GE, Barnes JM. Evaluation of Drug Activities. In: Pharmacometrics, Lawrence DR, Bacharach AL, Eds., Vol. 1. Academic Press: New York; 1964.

13. Robertson RP, Harmon J, Tran PO, Tanaka Y, Takahashi H. Glucose toxicity in beta-cells: type 2 diabetes, good radicals gone bad, and the glutathione connection. Diabetes 2003;52:581-7.

14. Robertson RP, Harmon J, Tran PO, Poitout V. Betacell glucose toxicity, lipotoxicity and chronic oxidative stress in type 2 diabetes. Diabetes 2004;53 (suppl 1):S119-24.

15. Janson J, Ashley RH, Harrison D, McIntyre S, Butler PC. The mechanism of islet amyloid polypeptide toxicity is membrane disruption by intermediate-sized toxic amyloid particles. Diabetes 1999;48:491-8.

16. Ghaisas MM, Malusare PV, Talele SG, Kshirsagar AD, Deshpande AD. Hypoglycemic And AntiHyperglycemic Study Of Combination Of Curcumin and Quercetin In Normoglycemic Rats. Inventi Impact: Planta Activa 2011; Article ID"Inventi:ppa/49/11", 2011.

17. Nolte MS. Pancreatic Hormones \& Antidiabetic Drugs. In: Basic and Clinical Pharmacology 11th ed., Katzung BG, Masters SB, Trevor AJ editors. Tata McGraw Hill Education private Limited: New Delhi; 2009:727-51.

18. Sharma HL, Sharma KK. Insulin, Oral Hypoglycemics and Other related agents. In: Principles of Pharmacology, 1st ed. Paras Medical Publishers: Hyderabad; 2007:642-56.

19. Sharma RK, Patki PS. Double-blind, placebocontrolled clinical evaluation of an Ayurvedic formulation (Gluco Care capsules) in non-insulin dependent diabetes mellitus. Journal of Ayurveda \& Integrative Medicine 2010;1:45-51.

20. Tripathi KD. Insulin, Oral Hypoglycemic Drugs and Glucagon. In: Essentials of Medical Pharmacology 6th ed. Jaypee Brothers Medical Publishers (P) Ltd.: New Delhi; 2008:254-74.

doi:10.5455/2319-2003.ijbcp20130312

Cite this article as: Leve ST, Banga H, Shankar P, Dixit RK. An experimental study of a novel combination of a herbal drug with an allopathic drug to evaluate the antihyperglycemic effect of irbesartan plus curcumin and comparison with glibenclamide. Int J Basic Clin Pharmacol 2013;2:182-6. 